\title{
INDICADORES MATERNOS DOS PARTOS ASSISTIDOS EM UM CENTRO DE PARTO NORMAL INTRA-HOSPITALAR
}

\section{MATERNAL INDICATORS OF ASSISTED DELIVERIES IN AN IN-HOSPITAL NORMAL DELIVERY CENTER}

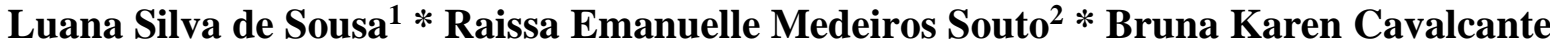 \\ Fernandes $^{3} *$ Cinthia Maria Gomes da Costa Escoto Esteche ${ }^{4}$ Ana Kelve de Castro \\ Damasceno $^{5} *$ Laura Pinto Torres de Melo ${ }^{6}$
}

\begin{abstract}
RESUMO
Objetivo: caracterizar os indicadores maternos dos partos assistidos por residentes de Enfermagem Obstétrica em um Centro de Parto Normal Intra-hospitalar. Métodos: estudo descritivo, documental e retrospectivo realizado utilizando o Livro de Registro de Partos assistidos pelos residentes, com total de 421 partos. Os dados foram processados no Programa Statical Package for the Social Sciences. Foram calculadas as frequências relativa/absoluta, teste qui-quadrado de Pearson e IC 95\%. Resultados: 54,2\% das mulheres eram multíparas, $89,1 \%$ teve gestação a termo, $95,5 \%$ foi admitida em fase ativa do trabalho de parto, $71,0 \%$ sofreu algum grau de laceração perineal, em $1,4 \%$ a episiotomia foi realizada. Os métodos não farmacológicos para alívio da dor mais utilizados foram respiração consciente, banho de aspersão, deambulação, massoterapia e bola suíça, respectivamente. Conclusão: a atuação dos residentes de enfermagem obstétrica possibilita a implementação de ações no ambiente de trabalho com foco na satisfação da mulher, na segurança do paciente e na qualidade da assistência.
\end{abstract}

Palavras-chave: Parto Humanizado; Enfermagem Obstétrica; Parto Normal.

\begin{abstract}
Objective: to characterize the maternal indicators of births attended by residents of Obstetric Nursing in an In-Hospital Normal Childbirth Center. Methods: descriptive, documentary and retrospective study carried out using the Birth Registration Book assisted by residents, with a total of 421 births. The data were processed using the Statical Package for the Social Sciences Program. Relative / absolute frequencies, Pearson's chi-square test and 95\% CI were calculated. Results: $54.2 \%$ of women were multiparous, $89.1 \%$ had full term pregnancies, $95.5 \%$ were admitted to active labor, $71.0 \%$ suffered some degree of perineal laceration, in $1.4 \%$ episiotomy was performed. The most widely used non-pharmacological methods for pain relief were conscious breathing, sprinkling bath, ambulation, massage therapy and Swiss ball, respectively. Conclusion: the performance of obstetric nursing residents enables the implementation of actions in the work environment with a focus on women's satisfaction, patient safety and quality of care. Keywords: Humanized Delivery; Obstetric Nursing; Normal Delivery.
\end{abstract}

\footnotetext{
${ }^{1}$ Enfermeira. Mestranda em Cuidados Clínicos em Enfermagem e Saúde (UECE). Especialista em Enfermagem Obstétrica (UECE). Residente de Enfermagem Obstétrica (UFC). Fortaleza, CE, Brasil. https://orcid.org/0000-0002-6203-0024 Email: lusilvasousa_@hotmail.com

2 Enfermeira. Residente de Enfermagem Obstétrica (UFC). Mestranda em Enfermagem pela Universidade da Integração Internacional da Lusofonia Afro-brasileira (UNILAB). Redenção, CE, Brasil. Orcid https://orcid.org/0000-0001-5995-2784 Email: raissadiogo@ hotmail.com

${ }^{3}$ Enfermeira. Docente da Universidade Federal do Piauí (UFPI), Doutora em Cuidados Clínicos em Enfermagem e Saúde (UECE). Floriano, PI, Brasil. Orcid https://orcid.org/0000-0003-2808-7526 Email: brunacavalcante@ufpi.edu.br

4 Enfermeira. Mestre em Enfermagem (UFC). Fortaleza, CE, Brasil. Orcid https://orcid.org/0000-0001-6958-3185 Email: cinthiaesteche@gmail.com

${ }_{5}^{5}$ Enfermeira. Mestre e Doutora em Enfermagem (UFC). Pós-Doutorado na University of British Columbia. Coordenadora do Programa de Residência em Enfermagem Obstétrica da Maternidade Escola Assis Chateaubriand (UFC). Docente Associada II da Graduação e da Pós-Graduação em Enfermagem da UFC. Fortaleza, CE, Brasil. Orcid https://orcid.org/0000-0003-4690-9327 Email: anakelve@hotmail.com

${ }^{6}$ Enfermeira. Especialista em Enfermagem Obstétrica (UECE). Mestre em Cuidados Clínicos em Enfermagem e Saúde (UECE).

Fortaleza, CE, Brasil. Orcid https://orcid.org/0000-0002-3030-2216 Email: lauraptmelo@gmail.com
} 


\section{ARTIGO ORIGINAL}

\section{INTRODUÇÃO}

$\mathrm{O}$ parto, até meados do século $\mathrm{XX}$, era um acontecimento íntimo e privativo, compartilhado apenas entre mulheres, como fenômeno fisiológico, cercado de significados culturais. Todavia, com a institucionalização do parto, mudanças significativas ocorreram nesse cenário ${ }^{(1)}$.

Até o final da década de 70, o modelo de assistência obstétrica era, predominantemente, caracterizado pela institucionalização do parto, centrado em atos médicos e no uso rotineiro de intervenções desnecessárias. Isto passou a ser questionado e criticado por movimentos feministas e de outros setores da sociedade ${ }^{(2)}$.

Frente a isso, os Centros de Parto Normal (CPN) foram criados com o intuito de resgatar a dignidade da mulher no processo parturitivo, visando exercer os princípios da humanização da assistência, valorizando o acolhimento, a escuta sensível e a relação respeitosa entre profissionais e usuárias ${ }^{(2)}$.

Assim, os CPN são considerados unidades de saúde que prestam atendimento humanizado e de qualidade, exclusivamente, ao parto normal sem distocias. Podem ser classificados em: CPN Intra-Hospitalar (CPNi) tipos I e II, bem como em CPN Perihospitalar (CPNp), sendo diferenciados pela estrutura física e organização do serviço ${ }^{(2-3)}$.

O CPNi Tipo II caracteriza-se por estar localizado nas dependências internas do estabelecimento hospitalar, possuir ambientes compartilhados com o restante da maternidade, como recepção, sala de exames, posto de enfermagem, sala de serviço e outros ambientes de apoio; garantir a permanência da mulher e do recém-nascido no quarto durante o pré-parto e parto, podendo, após o puerpério imediato, serem transferidos para o alojamento conjunto $^{(3)}$.

A criação dos CPN, sob a responsabilidade de enfermeiros obstétricos, desencadeou movimentos políticos e sociais questionadores da competência desses profissionais na atenção à mulher e ao recémnascido e das suas responsabilidades frente aos possíveis danos decorrentes da assistência perinatal $^{(1)}$.

A atuação e a responsabilidade dos enfermeiros obstetras na assistência segura às gestantes, parturientes e recém-nascidos nos serviços de obstetrícia e Centros de Parto Normal são normatizadas pelo Conselho Federal de Enfermagem, por meio da Resolução do Conselho Federal de Enfermagem $-n^{\circ} 516 / 2016$ - alterada pela Resolução no 524/2016(4).

A atenção prestada e a forma de relacionamento entre parturientes e enfermeiros obstetras, incluindo também os residentes, são fundamentais frente à humanização do parto e nascimento. Nos CPN, o acompanhamento contínuo por esses profissionais, embasado nas políticas públicas de saúde, fazendo uso das tecnologias apropriadas, necessárias e fundamentadas 


\section{ARTIGO ORIGINAL}

cientificamente, gera nas mulheres satisfação e segurança com o cuidado ofertado ${ }^{(5)}$.

Nessa perspectiva, a utilização de indicadores que avaliam a assistência de enfermagem é essencial para o gerenciamento dos serviços de saúde, pois permite o monitoramento dos resultados do cuidado ofertado e proporciona a identificação de necessidades de intervenções para melhoria do desempenho, produtividade e qualidade dos serviços ${ }^{(6)}$.

Ademais, a atuação da residência em enfermagem obstétrica vem se consolidando na assistência ao parto e nascimento de modo a garantir a qualificação da atenção obstétrica e neonatal, levando à redução das intervenções desnecessárias, ao respeito ao parto como um processo fisiológico e à integralidade do cuidado.

Assim, objetivou-se caracterizar os indicadores maternos dos partos assistidos por residentes de Enfermagem Obstétrica em um Centro de Parto Normal Intra-hospitalar. Nesse sentido, buscou-se responder a seguinte pergunta norteadora: quais os indicadores maternos dos partos assistidos por residentes de Enfermagem Obstétrica em um Centro de Parto Normal Intra-hospitalar?

\section{MÉTODOS}

Estudo descritivo, documental e retrospectivo, realizado no $\mathrm{CPNi}$ tipo II, localizado no Centro Obstétrico de uma maternidade pública de nível terciário, situada em Fortaleza, Ceará. Esta é composta por 8 suítes pré-parto, parto e pós-parto e atende uma média de 2041 partos vaginais ao ano. A equipe de enfermagem era composta por 11 enfermeiros obstetras, 19 técnicos de enfermagem, 6 auxiliares de enfermagem. Além desses, o serviço recebe, anualmente, 12 residentes de enfermagem obstétrica que são distribuídos por todos os setores do bloco materno-neonatal. No CPN, sempre ficam dois residentes em escalas organizadas de modo que todos os turnos sejam preenchidos, geralmente um residente do primeiro ano em dupla com um do segundo ano, pois possui mais experiência.

Fizeram parte do estudo 421 participantes, que atenderam ao seguinte critério de inclusão: preenchimento completo das informações no Livro de Registro de Partos. Esse livro é utilizado desde a primeira turma de residência como um documento para registrar os partos exclusivamente realizados pelos residentes, sendo validado pelo preceptor (enfermeiro ou médico) que acompanhou o processo de parturição com o residente e, posteriormente, pela coordenação. São anotadas as seguintes informações: data, iniciais do nome da paciente, idade, paridade, idade gestacional, diagnóstico na admissão, dilatação na admissão, métodos não farmacológicos para alívio da dor, laceração, grau da laceração, sutura, episiotomia/episiorrafia, apgar, peso, estatura, contato pele a pele e assinatura do residente e 


\section{ARTIGO ORIGINAL}

preceptor(a). Foram excluídos os registros com informações ilegíveis.

A coleta de dados foi realizada nos meses de setembro e outubro de 2018 por meio de um instrumento, elaborado pelos próprios autores, com as variáveis obstétricas: idade materna, paridade, idade gestacional, dilatação cervical na admissão, integridade perineal, utilização de métodos não farmacológicos para alívio da dor durante trabalho de parto e parto - a partir de todas as informações obtidas no Livro de Registro de Partos assistidos pelos residentes de julho de 2016 a julho de 2018, que foi o período em que estavam vinculados ao Programa de Residência em Enfermagem Obstétrica.

Os dados coletados foram processados no Programa Statical Package for the Social Sciences (SPSS) versão 20.0. Foram calculadas as frequências relativa, absoluta, média e desvio padrão. Para verificar a existência de associação entre duas variáveis categóricas, utilizou-se o teste Qui-quadrado de Pearson nos casos em que não houve frequência esperada menor que 5 em $20 \%$ ou mais das cédulas e com intervalo de confiança de $95 \%$. Em seguida, os dados foram apresentados em tabelas e analisados segundo a literatura pertinente ao tema em pesquisas internacionais e nacionais.

A pesquisa seguiu os princípios éticos que regem a pesquisa envolvendo seres humanos e foi aprovada pelo Comitê de Ética em Pesquisa da Maternidade Escola Assis Chateaubriand com Parecer $\mathrm{n}^{\circ}$ 1.684.564/2016 (Certificado de Apresentação para Apreciação Ética $\mathrm{n}^{\circ}$ 58294216.0.0000.5050). Foi assinado o Termo de Fiel depositário por se tratar de pesquisa com fontes secundárias.

\section{RESULTADOS}

A idade das mulheres que participaram do estudo variou entre 13 a 43 anos, com média de 24 anos e desvio padrão de $\pm 6,36$. A Tabela 1 apresenta os resultados relacionados às características obstétricas das mulheres atendidas por residentes de enfermagem obstétrica.

Tabela 1 - Distribuição dos indicadores maternos de parturientes atendidas por residentes de enfermagem obstétrica no período de jul/2016 a jul/2018, MEAC. Fortaleza, CE, Brasil, 2020.

\begin{tabular}{lc}
\hline Variáveis & n (\%) \\
\hline Paridade & $193(45,8)$ \\
Nulípara & $228(54,2)$ \\
Multípara & \\
Idade gestacional (semanas) & \\
https://doi.org/10.31011/reaid-2021-v.95-n.34-art.1067 Rev Enferm Atual In Derme v. 95, n. 34, 2021 e-021068
\end{tabular}


Dilatação cervical na admissão $(\mathrm{cm})$

$$
\begin{aligned}
& \leq 3 \\
& \geq 4
\end{aligned}
$$

Integridade perineal

Sem trauma ou sem sutura

Laceração

Grau $1^{\text {a }}$

Grau $2^{\mathrm{a}}$

Grau $3^{\mathrm{a}}$

Episiotomia

$6(1,4)$

Fonte: Dados da pesquisa, 2018.

Observou-se que mais da metade $(54,2 \%)$ das participantes da pesquisa foram multíparas. A maioria $(89,1 \%)$ apresentava gestação a termo e dilatação cervical na admissão maior ou igual a quatro centímetros $(95,5 \%)$. Percebe-se que $10,9 \%$ dos partos foram prematuros e $4,5 \%$ das mulheres foram admitidas na fase latente do trabalho de parto.

Percebeu-se que, em relação à integridade perineal, grande parte $(71,0 \%)$ apresentou algum grau de laceração, sendo $31,8 \%$ de primeiro grau, $37,1 \%$ de segundo grau e $2,1 \%$ de terceiro grau. Em

contrapartida, houve necessidade de episiotomia em pequena parcela $(1,4 \%)$ do estudo.

No tocante aos métodos não farmacológicos para alívio da dor mais utilizados nas parturientes (Tabela 2), observou-se que a maioria $(86,6 \%)$ utilizou as técnicas de respiração consciente, $62,9 \%$ o banho de aspersão, quase a metade $(50,3 \%)$ a deambulação, $45,3 \%$ a massoterapia e $42,2 \%$ a bola suíça. Estes foram os métodos não farmacológicos para alívio da dor mais prevalentes no estudo.

Tabela 2 - Distribuição dos métodos não farmacológicos para o alívio da dor utilizados nas parturientes no período de jul/2016 a jul/2018, MEAC. Fortaleza, CE, Brasil, 2020.

\section{Métodos não farmacológicos de alivio da dor*}

$$
\text { n }(\%)
$$

Aromaterapia

Banho de aspersão $265(62,9)$

Bola $178(42,2)$ 
Cavalinho

$110(26,1)$

Deambulação

$212(50,3)$

Balanceio pélvico

$86(20,4)$

Penumbra

Respiração consciente

Massagem

$191(45,3)$

Musicoterapia

$45(10,6)$

*Variável admite mais de uma opção

Fonte: Dados da pesquisa, 2018.

Ressalta-se que as pacientes utilizaram uma ou mais opções dos métodos mostrados na Tabela 2. No tocante à Tabela 3, esta apresenta os resultados acerca da associação entre paridade e dilatação na admissão e a ocorrência ou não de laceração perineal.

Tabela 3 - Distribuição dos dados de laceração e dilatação na admissão segundo a paridade das parturientes no período de jul/2016 a jul/2018, MEAC. Fortaleza, CE, Brasil, 2020.

\begin{tabular}{lcccc}
\hline \multirow{2}{*}{ Características } & \multicolumn{2}{c}{ Paridade } & $\begin{array}{c}\text { Intervalo de } \\
\text { Confiança (95\%) }\end{array}$ & $\begin{array}{c}\text { P- } \\
\text { valor* }\end{array}$ \\
\cline { 2 - 3 } & Nulíparas & Multíparas & \\
\cline { 2 - 3 } Laceração & $\mathbf{n}(\%)$ & $\mathbf{n}(\%)$ & $(1,89-4,72)$ & 0,000 \\
Sim & $159(53,4)$ & $139(46,6)$ & & \\
Não & $34(27,6)$ & $89(72,4)$ & & \\
Dilatação na Admissão & & & $(1,02-3,05)$ & 0,039 \\
$\geq \mathbf{3 c m}$ & & & \\
Sim & $170(48,0)$ & $184(52,0)$ & & \\
Não & $23(34,3)$ & $44(65,7)$ & & \\
\hline
\end{tabular}

*Teste Qui-quadrado de Pearson

Fonte: Dados da pesquisa, 2018.

Percebeu-se, portanto, que houve associação estatisticamente significante entre laceração e paridade $\left(\mathrm{x}^{2}: 23,186 ; \mathrm{p}: 0,000\right)$, https://doi.org/10.31011/reaid-2021-v.95-n.34-art.1067 Rev Enferm Atual In Derme v. 95, n. 34, 2021 e-021068

com maior prevalência em nulíparas. No tocante a associação entre a dilatação $\geq 3 \mathrm{~cm}$ na admissão e paridade, houve significância (c) (i) 


\section{ARTIGO ORIGINAL}

estatística e as nulíparas chegaram ao serviço com maior dilatação do que as multíparas $\left(\mathrm{x}^{2}\right.$ : 4,255; p: 0,039).

\section{DISCUSSÃO}

$\mathrm{O}$ perfil das parturientes deste estudo foi semelhante ao já descrito em outra pesquisa que analisou os partos normais realizados num hospital de ensino de Cuiabá, em que a idade das mulheres variou entre $12 \mathrm{e}$ 45 anos, sendo a média 24,6 anos ${ }^{(7)}$.

Em relação à paridade, mais da metade era multípara. Quanto à idade gestacional, grande parte eram gestações a termo, havendo, ainda, partos prematuros. Estes achados foram semelhantes ao estudo supracitado em que um número significativo de partos decorreu de gestações a termo $(88 \%)$ seguidas das gestações pré-termo $(11,4 \%)^{(7)}$.

Constatou-se a admissão de um grande quantitativo de mulheres na fase ativa do trabalho de parto na ocasião da internação hospitalar. Sabe-se que a admissão precoce aumenta o tempo de internação da parturiente, submetendo-a ao ambiente hospitalar sem necessidade e potencializando o número intervenções desnecessárias ${ }^{(8)}$.

Em relação à integridade perineal, as parturientes tiveram partos sem lacerações ou sem sutura, porém mais da metade apresentou algum grau de laceração, em sua maioria com extensão até segundo grau, com 2,1\% atingindo terceiro grau.
Estes achados tem semelhança com resultados de outro estudo realizado com partos acompanhados por enfermeiros obstetras anotados no livro de registros, no Rio de Janeiro, em que $36,4 \%$ permaneceram com períneo íntegro, havendo apenas um caso de laceração perineal grave ${ }^{(9)}$.

Apesar de toda a assistência prestada durante o trabalho de parto, lesões perineais ainda são prevalentes e podem levar a sintomas fisiológicos, como dor, ardor, vermelhidão e edema, gerados pelo processo de cicatrização, além de complicações patológicas, como distúrbios do assoalho pélvico $^{(10)}$.

Revisão sistemática que objetivou estudar a sutura em laceração de primeiro e segundo graus encontrou que as suturas em lacerações de primeiro grau podem estar mais associadas a problemas perineais e mudanças na realização de atividades durante $o$ puerpério imediato do que aquelas que não foram suturadas ${ }^{(11)}$.

Houve necessidade de episiotomia em pequena parcela das mulheres, isto mostra que as práticas de assistência dos residentes em enfermagem obstétrica estavam de acordo com as recomendações propostas pela World Health Organization para uma experiência positiva no parto, em que não existe uma taxa definida acerca da realização da episiotomia, mas afirma que esse procedimento não deve ser feito rotineiramente ${ }^{(12)}$. 


\section{ARTIGO ORIGINAL}

Assim, é imprescindível a atuação do enfermeiro obstetra na adoção de ações que proporcionem o manejo adequado para a redução dos problemas perineais apresentados pelas mulheres no pós-parto vaginal. Isto pode resultar em melhoria na execução das atividades habituais e das necessidades fisiológicas $^{(10)}$.

A atuação do enfermeiro obstetra é estratégica, tendo papel fundamental na qualificação dos serviços de saúde e na assistência à mulher no processo de parturição. Esse profissional torna-se indispensável no acompanhamento da parturiente, pois a estimula a assumir seu papel de protagonista, sendo capaz de utilizar estratégias transformadoras do ambiente em que atua, traduzindo a ciência que aprendeu em moldes humanistas, levando em conta os direitos da mulher à maternidade segura e $\operatorname{prazerosa}^{(8,13)}$.

A complexidade dos fatores que cercam o cenário do parto envolve questionamentos que vão desde a qualidade da atenção obstétrica até o significado da parturição. Estudos pregressos, a respeito das percepções de profissionais e usuárias, revelaram que a humanização no parto e no nascimento abrange aspectos estruturais, atitude ética dos profissionais, comunicação interpessoal efetiva, envolvimento e respeito mútuo entre profissional-parturiente-familiares, perpassando desde o acolhimento à alta ${ }^{(14)}$.
A assistência de enfermagem obstétrica humanizada deve ser conduzida com sensibilidade, respeito e dignidade, criando uma atmosfera acolhedora, com posturas profissionais e institucionais que rompem com o modelo tradicional de impessoalidade e violência imposta às parturientes. Humanizar o parto é respeitar e criar condições favoráveis para o todo - eixos espirituais, psicológicos e biológicos dos seres humanos envolvidos - por meio de procedimentos comprovadamente benéficos para o binômio mãe-filho, evitando intervenções desnecessárias e estabelecendo relações baseadas em princípios éticos ${ }^{(15)}$.

A utilização de métodos não farmacológicos para alívio da dor durante o trabalho de parto e parto reforça a preocupação dos profissionais envolvidos na humanização da assistência em oferecer conforto e apoio às parturientes no enfrentamento da dor. A satisfação da mulher com seu parto não relaciona-se apenas à ausência da dor, mas às condições oferecidas para o seu enfrentamento ${ }^{(16)}$.

Além disso, quando os profissionais reconhecem e respeitam a fisiologia do parto, usam essas técnicas à disposição das mulheres para elas escolherem o que preferirem ou acharem melhor, de acordo com sua necessidade e vontade, promovendo, assim, a liberdade e a autonomia das parturientes com seus corpos ${ }^{(1,16)}$. 
Os métodos mais utilizados pelos como a corresponsabilização pela assistência, residentes de enfermagem obstétrica em sua prática foram: técnicas de respiração consciente banho de aspersão, deambulação, massoterapia e bola suíça. Este achado corrobora com estudo realizado em hospital público da região Sul em que as mulheres tiveram mais acesso a esses métodos; em contrapartida, a mesma pesquisa indicou que essas experiências ainda são pontuais e que dependem da filosofia assistencial da instituição de saúde ${ }^{(17)}$.

Evidenciou-se, neste estudo, que todas as mulheres assistidas utilizaram um ou mais métodos não farmacológicos para alívio da dor, o que vai ao encontro de outra pesquisa realizada em Porto Alegre, onde foi feita análise de 232 prontuários de parturientes que tiveram parto vaginal conduzido por enfermeiros obstetras. Os métodos não farmacológicos de alívio da dor utilizados no trabalho de parto e no parto foram: deambulação $(79,2 \%)$, banho $(73,1 \%)$, massagem (60,0\%), liberdade de posição $(58,8 \%)$, aromaterapia $(46,9 \%)$, bola suíça $(42,0 \%)$, rebozo $(12,7 \%)$, escalda-pés $(2,4 \%)$ e musicoterapia $(2,0 \%)^{(18)}$.

Vale ressaltar que o uso dessas tecnologias foi ofertado de maneira educativa, elencando os objetivos e modo de realização de cada uma. Além disso, a parturiente teve o direito de aceitar ou não as condutas, respeitando suas vontades. Percebe-se, desse modo, o fortalecimento da autonomia, bem visto que a mulher é orientada a participar das decisões relacionadas ao seu trabalho de parto e parto ${ }^{(19)}$.

Em relação aos dados que demonstraram associação estatisticamente significativa entre laceração e paridade, pesquisa internacional corrobora com o achado de que a primiparidade pode ser um fator de risco à laceração perineal, bem como afirma que as nulíparas chegam à maternidade com dilatação mais avançada que as multíparas $^{(20)}$.

Ademais, o manejo adequado que envolva a mitigação de problemas relacionados a lacerações perineais torna-se essencial dentro da atuação da enfermagem obstétrica. Isto pode acarretar em maior satisfação das mulheres com os cuidados $\operatorname{ofertados}^{(10)}$.

Assim, a propagação e a implementação das boas práticas de assistência ao parto e nascimento torna-se efetiva com a atuação da residência em enfermagem obstétrica, além da coesão entre os trabalhadores do serviço e a organização como um todo em prol do cuidado de excelência.

\section{CONCLUSÃO}

Conclui-se que a maioria das mulheres era multípara, teve gestação a termo, foi admitida em fase ativa do trabalho de parto, sofreu algum grau de laceração envolvendo lacerações com extensão até segundo grau. Os 


\section{ARTIGO ORIGINAL}

métodos não farmacológicos para alívio da dor mais utilizados foram as técnicas de respiração consciente, o banho de aspersão, a deambulação, a massoterapia e a bola suíça, respectivamente. A laceração e dilatação na admissão $\geq 3 \mathrm{~cm}$ teve associação estatisticamente significante com a paridade, com prevalência em nulíparas.

As limitações deste estudo estiveram relacionadas ao preenchimento inadequado do Livro de Registros e à ausência de outras variáveis importantes, como dados sociodemográficos, número de consultas prénatal, realização de amniotomia, uso da ocitocina, da analgesia, do partograma, posição no parto, dentre outras. Além disso, a utilização do método descritivo impossibilitou a identificação de relações de causa e efeito entre as variáveis analisadas.

Todavia, os dados desta pesquisa apresentam potencial para contribuir com desenvolvimento e/ou aprimoramento de programas de atenção à saúde da parturiente, bem como de gestão dos serviços, que devem considerar os principais indicadores maternos e proporcionar cuidados condizentes com as reais necessidades desta população.

Os resultados mostram que a atuação dos residentes de enfermagem obstétrica dentro dos Centros de Parto Normal possibilita a implementação de ações no ambiente de trabalho com foco na segurança, na satisfação da mulher e na qualidade da assistência obstétrica.

\section{REFERÊNCIAS}

1. Vargens OMC, Silva ACV, Progiant JM. The contribution of nurse midwives to consolidating humanized childbirth in maternity hospitals in Rio de Janeiro-Brazil. Esc Anna Nery [Internet]. 2017 [acesso em 20 fev 2021]; 21(1):e20170015. Disponível em: https://www.scielo.br/scielo.php?pid=S141481452017000100215\&script=sci_arttext\&tlng =en

2. Lima MFG, Pequeno AMC, Rodrigues DP, Carneiro C, Morais APP, Negreiros FDS. Developing skills learning in obstetric nursing: approaches between theory and practice. Rev. Bras. Enferm [Internet]. 2017 Oct [cited 2021 May 04]; 70(5):1054-1060. Available from: http://www.scielo.br/scielo.php?script=sci_art text\&pid=S0034$71672017000501054 \& \operatorname{lng}=\mathrm{en}$.

3. Brasil. Ministério da Saúde (BR). Portaria $\mathrm{n}^{\mathrm{o}}$ 11, 07 de janeiro de 2015. Redefine as diretrizes para implantação e habilitação de Centro de Parto Normal (CPN), no âmbito do Sistema Único de Saúde (SUS), para o atendimento à mulher e ao recém-nascido no momento do parto e do nascimento, em conformidade com o Componente Parto e Nascimento da Rede Cegonha, e dispõe sobre os respectivos incentivos financeiros de investimento, custeio e custeio mensal. Brasília: Ministério da Saúde; 2015. Disponível em: 


\section{ARTIGO ORIGINAL}

http://bvsms.saude.gov.br/bvs/saudelegis/gm/ 2015/prt0011_07_01_2015.html\#: :text=1\% C2\%BA\%20Esta\%20Portaria\%20redefine\%2 0as,Cegonha\%2C\%20e\%20disp\%C3\%B5e\% 20sobre\%20os

4. Conselho Federal de Enfermagem. Resolução COFEN no 516/2016 - alterada pela Resolução COFEN no 524/2016, de 24 de junho de 2016 [Internet]. 2016 [citado 2020 mar 19]. Disponível em: http://www.cofen.gov.br/resolucao-cofen-no05162016_41989.html

5. Rocha BD, Zamberlan C, Backes DS, Freitas HMB, Costenaro RGS, Bordignon, J.S. Exchange of professional knowledge between residencies in obstetric nursing. Rev baiana enferm [Internet]. 2017 [acesso em 10 fev 2021]; 31(1):e181432017. Disponível em: https://periodicos.ufba.br/index.php/enfermag em/article/view/18143

6. Oliveira DMC, José FSR, Martins GAN, Matos AMR. Indicadores de qualidade: papel do enfermeiro para evitar iatrogenias obstétricas. Brazilian Journal of Health Review [Internet]. 2021 [acesso em 04 mai 2021]; 4(2):6402-6410. Disponível em: https://www.brazilianjournals.com/index.php/ BJHR/article/view/26944

7. Medeiros RMK, Teixeira RC, Nicolini AB, Alvares AS, Corrêa ACP, Martins DP. Cuidados humanizados: a inserção de enfermeiras obstétricas em um hospital de ensino. Rev Bras Enferm [Internet]. 2016 [acesso em 10 jan 2021]; 69(6):1029-36.
Disponível

https://www.scielo.br/pdf/reben/v69n6/00347167-reben-69-06-1091.pdf

8. Reis TR, Zamberlan C, Quadros JS, Grasel JT, Moro ASS. Enfermagem obstétrica: contribuições às metas dos Objetivos de Desenvolvimento do Milênio. Rev Gaúcha Enferm [Internet]. 2015 [acesso em 02 mar 2021]; 36(Spe):94-101. Disponível em: https://www.scielo.br/pdf/rgenf/v36nspe/0102 -6933-rgenf-36-spe-0094.pdf

9. Reis CSC, Souza DOM, Nogueira MFH, Progianti JM, Vargens OMC. Analysis of births attended by nurse midwives under the perspective of humanization of childbirth. $\mathbf{J}$ Res Fundam Care Online [Internet]. 2016 [acesso em 03 mar 2021]; (8)4:4972-79. Disponível em: http://www.seer.unirio.br/index.php/cuidadof undamental/article/view/3966/pdf

10. Santos LM, Santos LMS, Brandão MM, Cerqueira EAC, Ramos MSX, Carvalho ESS. Associação entre perineorrafia e problemas perineais, atividades habituais e necessidades fisiológicas afetadas. Rev Cuid [Internet]. 2018 [acesso em 03 mar 2021]; 9(2):2233-44. Disponível em: https://revistas.udes.edu.co/cuidarte/article/vi ew/530

11. Souza MRT, Farias LMVC, Ribeiro GL, Coelho TS, Costa CC, Damasceno AKC. Factors related to perineal outcome after vaginal delivery in primiparas: a crosssectional study. Rev. esc. enferm. USP 
[Internet]. 2020 [cited 2021 May 04];

54:e03549. Available from:

http://www.scielo.br/scielo.php?script=sci_art

text\&pid=S0080-

$62342020000100404 \& \operatorname{lng}=\mathrm{en}$.

12. World Health Organization.

Recommendations: intrapartum care for a positive childbirth experience. Geneva: World Health Organization; 2018. Disponível em: https://apps.who.int/iris/bitstream/handle/106 65/260178/9789241550215eng.pdf;jsessionid=EB689249D2567D283836 4D4B9C30EC8A? sequence $=1$

13. Dias EG, Monção PR, Cerqueira NC, Souza MAS. Assistência de Enfermagem no parto normal em um hospital público de Espinosa, Minas Gerais, sob a ótica da puérpera. Rev Interdisc [Internet]. 2016 [citado 2020 mar 19]; (9)2: 38-48. Disponível em:

https://revistainterdisciplinar.uninovafapi.edu. br/index.php/revinter/article/view/910/pdf_31 0

14. Rocha FAA, Fontenele FMC, Carvalho IR, Rodrigues IDCV, Sousa RA, Ferreira Júnior AR. Care during labor and birth: mothers' perception. Rev Rene [Internet]. 2015 [acesso em 20 fev 2021]; (16)6:782$789 . \quad$ Disponível em: http://periodicos.ufc.br/rene/article/view/2858 $/ 2221$

15. Baggio MA, Pereira FC, Cheffer MH, Machineski GG, Reis ACE. Significados e experiências de mulheres que vivenciaram o parto humanizado hospitalar assistido por enfermeira obstétrica. Rev baiana enferm. [Internet]. 2021 [ acesso em 04 de mai 2021]; 35: e42620. Disponível em: https://cienciasmedicasbiologicas.ufba.br/inde x.php/enfermagem/article/viewFile/42620/24 559

16. Boryri $\mathrm{T}$, Noori NM, Teimori A, Yaghonobinia F. The perception of primiparous mothers of comfortable resources in labor pain (a qualitative study). Iran J Nurs Midwifery Res. 2016; (21)3:239-46. Disponível em: https://www.ijnmrjournal.net/article.asp?issn $=1735$ -

9066; year $=2016$; volume $=21 ;$ issue $=3$; spage $=2$

39 ; epage $=246$; aulast $=$ Boryri

17. Scarton J, Ressel LB, Siqueira $\mathrm{HCH}$, Rangel RF, Tolfoet $\mathrm{F}$ et al. Care practices in normal birth: the experience of primiparous women. Rev Fund Care Online. [Internet]. 2018 [acesso em 04 de mai 2021]; 10(1):1724 Disponível em: http://www.seer.unirio.br/index.php/cuidadof undamental/article/view/5918/pdf

18. Lehugeur D, Strapasson MR, Fronza E. Non-pharmacological management of relief in deliveries assisted by an obstetric nurse. Rev Rnferm UFPE on line [Internet]. 2017 [acesso em 23 fev 2021]; 11(12):4929-37. Disponível em:

https://periodicos.ufpe.br/revistas/revistaenfer magem/article/view/22487/25308 
19. Soares YKC, Melo SSS, Guimarães TMM, Feitosa VC, Gouveia MTO. Satisfaction of puerperal women attended in a normal birth center. Rev Enferm UFPE on line [Internet]. 2017 [acesso em 03 mar 2021]; 11 (Supl. 11):4563-73. Disponível em: https://periodicos.ufpe.br/revistas/revistaenfer magem/article/view/231195/25186

20. Pergialiotis V, Bellos I, Fanaki M, Vrachnis N, Doumouchtsis SK. Risk factors for severe perineal trauma during childbirth: an update meta-analysis. Eur $\mathbf{J}$ Obstet Gynecol Reprod Biol [Internet]. 2020 [acesso em 03 mar 2021]; 247:94-100. Disponível em: https://www.ejog.org/article/S0301-

2115(20)30093-2/fulltext

Submissão: 2021-03-26

Aprovado: 2021-05-11 